\title{
Cuidar de quem cuida: eficácia de um programa de inteligência emocional para enfermeiros* $^{*}$
}

\author{
Caring for those Who Care: Effectiveness of an Emotional Intelligence Program for Nurses \\ Cuidar de quien cuida: eficacia de un programa de inteligencia emocional para enfermeros
}

Ana Filipa Silva Pereira

Universidade Fernando Pessoa, Portugal

ORCID: https://orcid.org/0000-0002-8337-2672

Ana Inês Santos Prior

Universidade Fernando Pessoa, Portugal

ORCID: https://orcid.org/0000-0001-6150-4681

\author{
Marlene Ferreira \\ Universidade Fernando Pessoa, Portugal \\ ORCID: https://orcid.org/0000-0003-4469-7527
}

\section{Carla Fonte $^{\text {a }}$}

Universidade Fernando Pessoa, Portugal

cfonte@ufp.edu.pt

ORCID: https://orcid.org/0000-0002-7280-2083
DOI: https://doi.org/10.11144/Javeriana.ie22.cpep

Recepção: 16 Abril 2020

Aprovação: 21 Setembro 2020

Publicação: 30 Dezembro 2020

\section{Resumo:}

Objetivo: Avaliar a eficácia de um programa de promoção da inteligência emocional, em enfermeiros em exercício de funções num hospital do Porto. Metodo: Estudo exploratório e quase-experimental, misto, com recurso à Escala de Inteligência Emocional de Wong e Law; Continuum de Saúde Mental- versão reduzida para adultos; Escala de Ansiedade, Depressão e Stress - 21 itens; Rahim Organizational Conflict Inventory. A amostra incluiu 6 enfermeiros, maioritariamente do sexo feminino, com idades compreendidas entre os 29 e os 35. Resultados: Os enfermeiros desenvolveram e aplicaram estratégias mais eficazes ao nível da autoconsciência, clarificação, autorregulação emocional, comunicação e gestão do conflito, permitindo um maior bem-estar social e emocional. Conclusões: $\mathrm{O}$ desenvolvimento de competências de Inteligência Emocional são essenciais na prestação de cuidados, permitindo a adoção de comportamentos adaptativos, a aprimoração de habilidades de gestão/regulação emocional promovendo o bem-estar, fulcrais para o processo de humanizar os cuidados.

Palavras-chave: Inteligência emocional, cuidados de saúde, humanização, enfermeiros.

\begin{abstract}
:
Objective: To evaluate the effectiveness of a program to promote emotional intelligence on nurses working on their regular functions in a hospital in Porto. Method: Exploratory quasi-experimental mixed study using as resources the Wong and Law Emotional Intelligence Scale; Mental Health Continuum - short version for adults; the Anxiety, Depression, and Stress Scale - 21 items; and the Rahim Organizational Conflict Inventory. The sample included 6 nurses, most of them female, between 29 and 35 years of age. Results: The nurses developed and applied more effective strategies at the self-awareness, clarification, emotional selfregulation, communication, and conflict management levels, allowing for greater social and emotional well-being. Conclusions: The development of Emotional Intelligence competencies is essential in the provision of care, enabling the adoption of adaptive behaviors, the improvement of management/emotional regulation skills for the promotion of well-being, which are central to the humanization process of care.
\end{abstract}

Keywords: Emotional intelligence, health care, humanization, nurses.

\section{Autor notes}

\footnotetext{
a Autora de correspondencia. Correo electrónico: cfonte@ufp.edu.pt
} 


\section{Resumen:}

Objetivo: Evaluar la eficacia de un programa de promoción de la inteligencia emocional, en enfermeros en ejercicio de sus funciones en un hospital do Porto. Método: Estudio exploratorio de corte casi-experimental, mixto, con recursos en la Escala de Inteligencia Emocional de Wong y Law; Continuum de Salud Mental- versión reducida para adultos; Escala de Ansiedad, Depresión y estrés - 21 items; Rahim Organizational Conflict Inventory. La muestra incluyó 6 enfermeros, mayoritariamente de sexo femenino, con edades comprendidas entre los 29 y los 35 años. Resultados: Los enfermeros desarrollaron y aplicaron estrategias más eficaces a nivel de la autoconsciencia, la clarificación, la autorregulación emocional, la comunicación y la gestión del conflicto, permitiendo un mayor bienestar social y emocional. Conclusiones: El desarrollo de competencias de Inteligencia Emocional son esenciales en la prestación de cuidados, permitiendo la adopción de comportamientos adaptativos, el perfeccionamiento de habilidades de gestión/ regulación emocional promoviendo el bienestar, centrales para el proceso de humanización de los cuidados.

Palabras clave: Inteligencia emocional, cuidados de salud, humanización, enfermeros.

\section{Introdução}

A inteligência emocional (IE) apresenta um papel preponderante nos contextos de saúde, proporcionando um equilíbrio emocional, facilitador na gestão das adversidades do processo de cuidar (1). Além disso, tem sido identificado como um elemento fundamental na otimização do desempenho profissional na regulação emocional, gestão do stress e diminuição dos níveis de burnout $(2,3)$.

Segundo Goleman (4), a IE refere-se à capacidade de percecionar, compreender, avaliar, expressar e reconhecer emoções do próprio e nos outros, promovendo o crescimento emocional e intelectual. Assim, alguns trabalhos sublinham que o treino de competências técnicas e psicossociais são fundamentais para a humanização dos cuidados de saúde, garantindo uma maior eficiência na prestação de serviços com efeitos diretos na organização $(1,2,3 ; 5,6)$.

O conceito de prática profissional na enfermagem passa por competências clínicas e pelo cuidado e relação com o doente (7). Deste modo, considerando o papel do enfermeiro e os desafios laborais que enfrentam (e.g. complexidade nas funções, o contacto com múltiplos intervenientes), implica, necessariamente, a exposição a fatores adversos, originando problemas de saúde mental (ansiedade, depressão, irritabilidade, fadiga, stress, alterações de sono, isolamento, conflitos interpessoais), com impacto na qualidade de vida, na postura do profissional e no seu bem-estar (social, subjetivo e psicológico) $(8,9,10)$. Também as características da organização (condições, relacionamentos interpessoais, salário, carga laboral), interferem na qualidade da prestação dos serviços, nos estados emocionais e no bem-estar (11).

Autores apontam que a aquisição de competências de IE visa uma otimização da autoconsciência emocional, traduzindo-se em habilidades de gestão de conflitos, autocontrolo e comunicação $(4,9,12)$. A capacidade de regular as emoções é relevante para a saúde mental, fortalecendo a atenção e a cognição, aumentando o bem-estar, a qualidade das relações sociais e o desempenho laboral (12). Desta forma, o ato de humanizar deve ser primordial no enfermeiro, recorrendo à empatia, espírito de interajuda e gestão emocional, tornando-o capaz de construir uma realidade mais consciente, respeitando a essência humana (2, $3,12,13,14)$.

Alguma literatura identifica a necessidade do treino de competências de IE, evidenciando que trabalhando este constructo se desenvolve maior acuidade emocional (1). Assim, estudos apontam para resultados positivos quando combinadas estas competências com a gestão de stress, permitindo uma manutenção da saúde e desenvolvimento de carreira, destacando que os enfermeiros que obtiveram maior formação, apresentam uma maior autoconsciência e autoperceção dos seus estados emocionais $(5,14)$. Neste sentido, o desenvolvimento de formação em IE permite o desenvolvimento de estratégias que tornam o processo de adaptação às adversidades mais acessível (1). Assim, criou-se um programa que procurou fomentar estas competências, melhorando o bem-estar, ambiente de trabalho e prática profissional. Procura-se com o presente estudo avaliar a eficácia de um programa de intervenção em grupo, de promoção da IE, em enfermeiros de um hospital do Porto. 


\section{Metodo}

A escolha da metodologia a utilizar foi realizada com base no objetivo do presente estudo. Assim, o desenho deste é de carácter exploratório, quase-experimental e misto. Esta escolha incidiu por ser uma abordagem que fornece resultados mais consistentes devido ao desenho de investigação ser mais robusto, possibilitando uma maior recolha de informações, maximizando a qualidade das conclusões retiradas, apresentando como principal vantagem a integração da informação, permitindo responder ao objetivo definido, com recursos a análises quantitativas e qualitativas (15). Especificamente, ao nível quantitativo, os dados foram introduzidos e tratados na base de dados criada no Statistical Package for Social Science (SPSS) versão 25. Para a determinação dos testes estatísticos, procedeu-se à análise exploratória, utilizando-se o teste de Kolmogorov-Smirnov para analisar a normalidade da distribuição das variáveis. Esta análise revelou a necessidade de utilização de testes não paramétricos, uma vez que a amostra não seguia uma distribuição aproximadamente normal, não ter sido escolhida aleatoriamente e ter uma reduzida dimensão (16). Salienta-se que, por se tratar de uma intervenção em grupo, o tamanho efetivo da amostra revelou-se adequado. Com o intuito de avaliar a existência de diferenças entre o pré e pós teste, optou-se pelo recurso ao teste de Wilcoxon (Z). Em todas as análises estatísticas, assumiu-se um p de 0,05 como valor de significância dos resultados dos testes e, 0,1 como resultados marginalmente significativos (16, 17). Quanto aos dados qualitativos, através dos relatos obtidos nos questionários, recorreu-se ao método da análise de conteúdo indutiva, que pressupõe que as categorias codificadas são derivadas diretamente dos dados de texto. Esta análise respeita três fases: preparação, organização e relato de resultados. Na primeira foram realizadas transcrições que resultaram em unidades de análise. Na segunda foi realizado um processo que incluí codificação aberta, criação de categorias e abstração, através de sub-categorias agrupadas com base nas suas semelhanças (categorias de $2^{\mathrm{a}}$ ordem). Na terceira, os resultados correspondem aos significados das sub-categorias que dão origem a uma descrição geral do tópico (categorias de $1^{\text {a }}$ ordem) (18).

Os participantes foram selecionados através de uma amostragem intencional, não probabilística (19), onde os enfermeiros foram elegidos pelas suas chefias, pelo critério de serem quem mais beneficiaria do programa. Como critérios de inclusão: a) ter mais de 18 anos; b) exercer funções há pelo menos 1 ano e c) prestar serviços na Unidade de Cuidados Continuados e da Unidade de Convalescença, Reabilitação e Manutenção. A amostra foi composta por 6 enfermeiros, maioritariamente do sexo feminino, com idades compreendidas entre os 29 e os 35 anos, solteiros, licenciados e a exercer funções há cerca de 8 anos (em média).

Foi solicitada autorização à Comissão de Ética da Universidade Fernando Pessoa, bem como o Consentimento Informado dos participantes, tendo sido assim cumpridos os procedimentos éticos considerados na "Declaração de Helsínquia" da Associação Médica Mundial. Não foi necessário realizar pedidos aos autores dos instrumentos, visto que estes se encontravam publicados e de livre acesso. Os procedimentos adotados junto dos participantes dividiram-se na recolha de dados, que ocorreu de forma individual e em dois momentos (antes da $1^{\text {a }}$ sessão -pré-teste e no final da $3^{\text {a }}$ sessão - pós-teste) e na aplicação do programa. Recorreu-se aos seguintes instrumentos: a) Questionário Sociodemográfico, b) Versão Portuguesa da Escala de Inteligência Emocional de Wong e Law (2002) - WLEIS-P, c) Continuum de Saúde Mental- versão reduzida para adultos (MHC-SF); d) Escala de Ansiedade, Depressão e Stress - EADS-21; e) Rahim Organizational Conflict Inventory - ROCI-II - Forma A, B e C e, f) Questionário de Avaliação da Sessão.

Quanto ao questionário sociodemográfico, este é composto por itens de identificação das características sócio demográficas da amostra (e.g. idade, escolaridade, estado civil, anos de funções). Relativamente a WLEIS-P, a sua versão original foi desenvolvida por Wong e Law em 2002 e foi validada para a população portuguesa por Rodrigues, Rebelo e Coelho em 2011 apresentando um alpha de 0,82 (20). É um instrumento de autorresposta, com 16 itens que avaliam as dimensões de IE (20): a) Avaliação e Expressão das próprias 
emoções; b) Avaliação e Reconhecimento das emoções nos outros; c) Regulação das emoções do próprio e d) Utilização das emoções para facilitar o desempenho. As dimensões incluem 4 itens, numa escala de tipo likert, desde 1 = "Discordo Fortemente" a 5 = "Concordo Fortemente" (21). No que respeita ao MHC-SF, este foi desenvolvido por Lamers e colaboradores em 2011, tendo sido validada para o contexto português por Fonte, Silva, Vilhena e Keyes em 2020 (22, 23, 24). É um questionário multidimensional, de autopreenchimento, composto por 14 itens, distribuídos por três dimensões: Bem-Estar Emocional, Bem-Estar Social e Bem-Estar Psicológico. Os entrevistados avaliam a frequência de cada sentimento no mês passado, numa escala tipo likert, desde 1- "Nunca" a 6 - "Todos os dias" (22). No que concerne às qualidades psicométricas, o estudo da validação do instrumento para a população portuguesa apresenta um alpha de 0,80 (24). A EADS-21 advém da Depression Anxiety Stress Scale - DASS criada por Lovibond e Lovibond em 1995. Foi traduzida e adaptada para a população portuguesa por Ribeiro, Honrado e Leal em 2004 e propõe-se medir os sintomas de ansiedade, depressão e stress $(24,25)$. Cada constructo previamente enunciado representa uma subescala, composta por 7 itens, perfazendo assim o total dos 21 . A subescala da ansiedade apresenta um alpha de 0,85; a subescala da depressão um alpha de 0,84 e, a de stress um alpha de 0,81 . As opções de resposta distribuem-se numa escala tipo Likert e variam entre 0 "Não se aplicou a mim" a 3 "Aplicou-se a mim, a maior parte das vezes", representando assim a severidade e frequência dos sintomas experienciados nos últimos 7 dias $(24,25)$. No que concerne ao ROCI-II, este foi construído por Rahim em 1983 e validado para a população portuguesa por Cunha, Moreira e Silva em 2003, com um alpha de 0,81 $(26,27)$. É um questionário de autopreenchimento que tem como objetivo conhecer os estilos de gestão de conflito que estão na base das relações interpessoais de Rahim e Bonoma (1979) (26). É composto por 28 itens, que se distribuem por 5 dimensões e que se baseiam na conceptualização dos cinco estilos de gestão do conflito interpessoal: integração, evitação, dominação, anuência e compromisso (27). Este instrumento desdobra-se em três formas distintas (A - relação com superiores; B-contacto com subordinados; $\mathrm{C}$ - relação entre parceiros ou colegas), com indicação de utilização de cada uma, de acordo com os níveis de hierarquia/relações estabelecidas nas organizações. A resposta ao questionário é feita numa escala de Likert, sendo que o 1 corresponde a "Discordo Fortemente" e o 5 a "Concordo Fortemente" (26, 27). Por último, o questionário de avaliação da sessão visa recolher a opinião dos participantes e é composto por duas partes (quantitativa e qualitativa). A primeira inclui 18 questões, numa escala de 1 (Mau) a 5 (Muito Bom) e divididas em três grupos: 1) a avaliação que o participante faz sobre os objetivos/conteúdos programáticos; 2) a avaliação do participante sobre o funcionamento da sessão e 3) a avaliação que o participante faz sobre o desempenho dos formadores. A segunda é composta por 3 questões de resposta aberta sobre a perceção da aprendizagem adquirida; a avaliação face às características da sessão/programa e sugestões indicadas para programas futuros.

O programa foi composto por 3 sessões com cerca de $3 \mathrm{~h}$ cada com as seguintes temáticas: a) Inteligência Emocional; b) Comunicação e c) Resolução de Conflito e foi implementado por três formadoras, investigadoras deste artigo e presentes em todas as sessões. Estas decorreram numa sala do hospital e com periodicidade mensal.

\section{Resultados: Análises Quantitativas}

De seguida apresenta-se os resultados significativos no pré e pós teste. 
TABELA 1.

Síntese dos resultados pré e pós teste

\begin{tabular}{lccc}
\hline & Resultados Pré-Teste & Resultados Pós-Teste & $\begin{array}{c}\text { Teste Wilcoxon- } \\
\text { Pré e Pós }(s i g)\end{array}$ \\
\cline { 1 - 2 } Instrumento & $M$ & $M$ & $\mathbf{0 , 0 8 ^ { * }}$ \\
\hline EADS Stress & $\mathbf{3 , 1 7}$ & $\mathbf{5 , 6 7}$ & $\mathbf{0 , 0 9 ^ { * }}$ \\
\hline ROCI(B) Anu. & $\mathbf{1 9 , 0 0}$ & $\mathbf{1 7 , 5 0}$ & $\mathbf{0 , 0 3}$ \\
\hline ROCI(C) Dom. & $\mathbf{1 5 , 8 3}$ & $\mathbf{1 4 , 3 3}$ & \\
\hline \multicolumn{5}{c}{ Fonte: elaboração própria. }
\end{tabular}

Após a aplicação do programa, observa-se um aumento, na dimensão Regulação das emoções na WLEIS$\mathrm{P}(\mathrm{M}=14,50 ; \mathrm{M}=15,50)$ e na dimensão do Bem-estar Social da $\mathrm{MHC}-\mathrm{SF}(\mathrm{M}=19,50 ; \mathrm{M}=20,83) . \mathrm{Na}$ EADS-21 verificam-se um aumento dos sintomas associados à ansiedade $(M=1,17 ; M=1,67)$, depressão $(M$ $=0,67 ; M=1,50)$ e stress $(M=3,17 ; M=5,67)$, este último marginalmente significativo $($ sig $=0,08>0,05)$. Em relação aos estilos de gestão de conflitos, nomeadamente na Relação com Superiores (ROCI-II Forma A) verifica-se uma diminuição no estilo de anuência $(M=20,76 ; \mathrm{M}=21,83)$, dominação $(M=14,67 ; \mathrm{M}=14,50)$ e evitação ( $M=20,00 ; M=19,17)$. Quanto à Relação com Subordinados (ROCI-II Forma B) verifica-se um aumento do estilo integração $(M=29,50 ; M=30,33)$ e compromisso $(M=15,17 ; M=15,33)$; observa-se uma diminuição da utilização do estilo dominação $(M=15,67 ; M=15,00)$, evitação $(M=19,83 ; M=18,99)$ e anuência $(M=19,00 ; M=17,50)$, este último com valores marginalmente significativos (sig = 0,09>0,05). $\mathrm{Na}$ Relação com Colegas (ROCI-II Forma C), identificam-se alterações na eleição dos estilos Integração (M $=28,67 ; M=30,17)$, Anuência $(M=15,83 ; M=16,33)$ e Compromisso $(M=14,67 ; M=15,33)$. Verifica-se um decréscimo no estilo evitação $(M=18,33 ; M=17,83)$, com alterações significativas no estilo Dominação $(M=15,83 ; M=14,33)$, notando-se uma diminuição na sua utilização (sig $=0,03<0,05)$.

\section{Análises Qualitativas}

A Tabela 2 sintetiza a perceção dos participantes face à aprendizagem adquirida. 
TABELA 2.

Perceção dos participantes face à aprendizagem adquirida

\begin{tabular}{|c|c|c|}
\hline Unid ades de Análise & $\begin{array}{l}\text { Categorias de } 2^{2} \\
\text { Ordem }\end{array}$ & $\begin{array}{l}\text { Categorias de } 1^{2} \\
\text { Ordem }\end{array}$ \\
\hline $\begin{array}{l}\text { "Promoveu a adoção de estratégias"-suj. } 1 \\
\text { "Permitiu refletir sobre as reações e estratégias } \\
\text { utilizadas"- Suj.2 } \\
\text { "Dotar de ferramentas práticas"-Suj. } 6 \\
\text { "Ajuda na prática profissional"-Suj. } 3 \\
\text { "Exercícios muito bons para assimilar a } \\
\text { informação"-Suj. } 4 \\
\text { "Sim, melhorar a relação com as outras } \\
\text { pessoas"-Suj. } 5 \\
\text { "Sim, ajudou a refletir sobre situações } \\
\text { problema"-Suj.1 } \\
\text { "Sim, permitiu melhorar atitudes"-Suj.1 } \\
\text { "Sim, permitiu aprender a importância da } \\
\text { comunicaç̃o nos conflitos"-Suj. } 1 \\
\text { "Sim, porque adequamos os estilos de } \\
\text { comunicação"-Suj. } 2 \\
\text { "Sim, porque adequamos os estilos de } \\
\text { lideranca"-Suj. } 2\end{array}$ & $\begin{array}{l}\text { Aquisição de } \\
\text { estratégias e } \\
\text { competências para } \\
\text { lidar e melhorar a } \\
\text { prática profissional }\end{array}$ & $\begin{array}{l}\text { Desenvolvimento de } \\
\text { conhecimentos e } \\
\text { competências } \\
\text { profissionais } \\
\text { fundamentais ao } \\
\text { contexto laboral }\end{array}$ \\
\hline $\begin{array}{l}\text { "Sim, permitiu aprender a importância da } \\
\text { autoconsciência" - Suj.1 } \\
\text { "Sim, permitiu aprender a importância do } \\
\text { autocontrol o" - Suj.1 } \\
\text { "Sim, enriqueceu o meu conhecimento sobre as } \\
\text { emoçōes" - Suj.2 }\end{array}$ & $\begin{array}{l}\text { Desenvolvimento de } \\
\text { competências } \\
\text { emocionais }\end{array}$ & $\begin{array}{c}\text { Promoção de } \\
\text { inteligência } \\
\text { emocional }\end{array}$ \\
\hline $\begin{array}{l}\text { "Sim, porque adequamos os estilos de gestão } \\
\text { de conflitos" - Suj. } 2 \\
\text { "Sim, aprendia resolver conflitos"-suj. } 5\end{array}$ & $\begin{array}{l}\text { Aqui sição de estratégias } \\
\text { de resol ução e gestão de } \\
\text { conflitos }\end{array}$ & \\
\hline
\end{tabular}

Fonte: elaboração própria.

Os participantes percecionam o desenvolvimento de conhecimentos e competências profissionais adquiridas como fulcrais ao contexto laboral (categoria de $1^{\text {a }}$ ordem), ressalvando a sua pertinência para lidar e melhorar a prática profissional (categoria de $2^{\mathrm{a}}$ ordem) através da aquisição de estratégias práticas passíveis de serem aplicadas a situações que possam emergir. Salientam uma maior consciência para as questões associadas à comunicação nas organizações (categoria de $2^{\mathrm{a}}$ ordem), nomeadamente na adoção de estilos de liderança e comunicação eficaz. Valorizam a promoção de IE (categoria de $1^{\text {a }}$ ordem), na ótica do desenvolvimento de competências emocionais e aquisição de estratégias de resolução e gestão de conflitos (categorias de $2^{a}$ ordem), permitindo uma maior autoconsciência, autocontrolo, capacidade de gestão emocional, capacidade de resolução de conflitos, adotando um estilo mais eficiente. A Tabela 3 abaixo remete para a avaliação dos participantes face às características do programa. 
TABELA 3.

Avaliação dos participantes face às características do programa

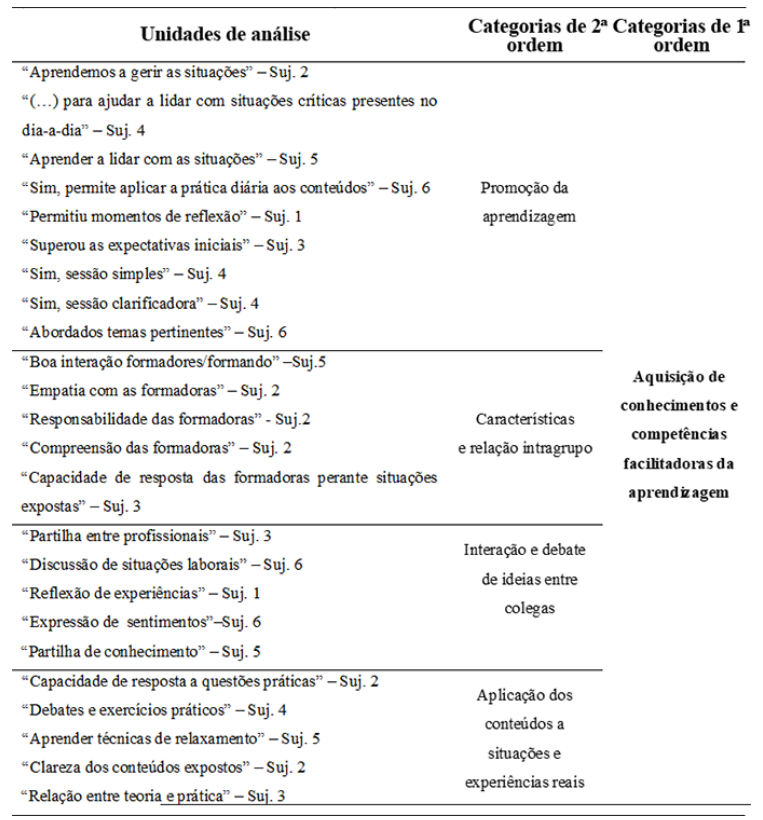

Fonte: elaboração própria.

O programa possibilitou a aquisição de conhecimentos e competências facilitadoras da aprendizagem (categoria de $1^{\text {a }}$ ordem), que se devem à promoção da mesma (categoria de $2^{a}$ ordem) e aplicação dos conteúdos a situações e experiências reais (categoria de $2^{\mathrm{a}}$ ordem) ajustada às necessidades diárias vivenciadas nos serviços hospitalares, proporcionadas através da existência de momentos de reflexão, exercícios práticos, clareza dos conteúdos expostos. Por outro lado, as características e relação intragrupo (categoria de $2^{\mathrm{a}}$ ordem), bem como a interação e debate de ideias entre colegas (categoria de $2^{a}$ ordem), foram um elemento facilitador à partilha de experiências, conhecimentos e entreajuda. Por fim, a Tabela 4 diz respeito às sugestóes indicadas pelos enfermeiros para programas futuros.

TABELA 4.

Sugestões indicadas

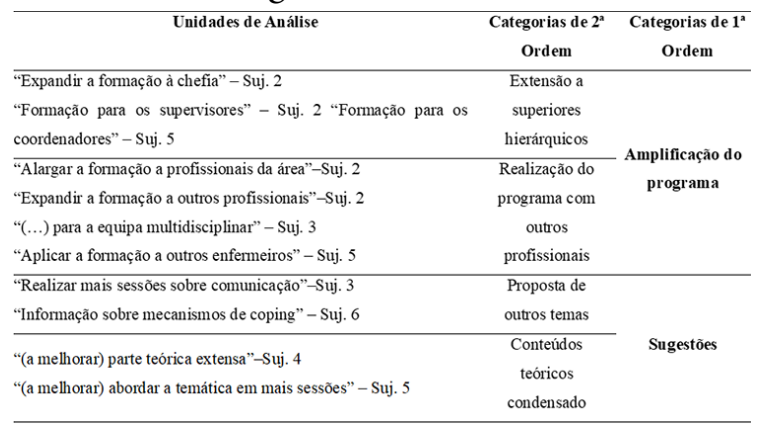

Fonte: elaboração própria.

Os participantes identificam a necessidade de amplificar o programa (categoria de $1^{\text {a }}$ ordem) junto de outros profissionais (da área e restante equipa multidisciplinar) e superiores hierárquicos (categorias de $2^{\mathrm{a}}$ ordem) - chefia, coordenadores e supervisores. Salvaguardam a necessidade de incluir outros temas, como os mecanismos de copping. Apontam para a necessidade de mais sessóes (sobre estes e outros temas) para que a parte teórica não seja tão condensada e extensa. 


\section{Discussão}

Com a implementação do programa, procurou-se contribuir para a promoção de competências emocionais e para a adoção de estratégias eficazes, fundamentais à prática profissional dos enfermeiros. Quanto às dimensões da IE, observa-se uma maior compreensão inerente à regulação dos estados emocionais, sendo este aspeto relevante para a adaptação a situações desafiantes e, para a saúde mental, visto fortalecer a atenção, a cognição e a criatividade, aumentando o bem-estar, a qualidade das relações sociais e o desempenho no trabalho (12).

No que respeita à saúde mental avaliada pelos diferentes tipos de bem-estar, verifica-se uma melhoria nas questóes sociais. Este aspeto vai de encontro com a literatura, devido à possibilidade de partilha, fortalecimento de relação e comparação de realidades vivenciadas por colegas, permitindo um aumento do sentimento de pertença, coesão grupal e social. Estes momentos são fulcrais ao crescimento profissional e pessoal, visto possibilitarem a troca de diálogo e experiências, minimizando as dificuldades comuns (12, 13, 14).

Relativamente às alterações ao nível de ansiedade, depressão e stress, estas podem atribuir-se a estados emocionais mais exaustivos, associadas ao aumento da fadiga, carga horária excessiva, falta de recursos humanos, rotatividade de horários, pouco tempo de descanso relatadas pelos participantes, provocando um aumento nos indicadores de nestes sujeitos, tal como referido pela literatura $(8,9,10,11)$.

Por fim, também em relação aos estilos de gestão de conflitos e, tendo em consideração os resultados, verifica-se uma maior consciência acerca dos mesmos e das suas implicações na relação com superiores, subordinados e colegas. Este aspeto demonstra uma maior compreensão de estilos mais saudáveis a utilizar para a gestão de conflito, recorrendo a uma resolução benéfica para os envolventes da situação conflituosa. Pode verificar-se, através da diminuição na utilização especificamente do estilo de anuência, comprovando o supracitado, ou seja, os profissionais começam a fazer uma melhor avaliação da situação de conflito e, facilmente entram no processo de negociação, em prol da defesa dos seus interesses, sem menosprezar os interesses dos outros (28). Isto significa que a autoconsciência emocional e a sensação de bem-estar, faz com que o individuo eleja os estilos mais benéficos para si, impactando na resolução de conflito (27).

\section{Conclusão}

Com esta investigação-ação conclui-se que o programa foi eficaz, na medida em que demonstra os benefícios que o treino de competências de IE apresenta nas funções do enfermeiro, nomeadamente na valorização do bem-estar minorando as complexidades inerentes a esta profissão. Especificamente, os resultados apontam que os objetivos foram alcançados, dotando estes profissionais de estratégias eficazes à perceção, autoconsciência, clarificação e autorregulação emocional, permitindo um ajustamento equilibrado aos desafios diários. Destacam-se a adoção e aplicação de ferramentas ao nível da comunicação e da gestão do conflito, que tornam a resolução de problemas mais consciente e eficaz junto de utentes, colegas e chefia, com impacto na saúde ocupacional. Consideramos que, apesar de se tratar de uma investigação quase-experimental, os seus resultados são uma mais-valia, tanto para a Enfermagem, como para a Psicologia, dado que a IE é uma habilidade inerente e impulsionadora do cuidado humano.

\section{Referências}

1. Raeissi P, Zandian H, Mirzarahimy T, Delavari S, Moghadam T, Rahimi G. Relationship between communication skills and emotional intelligence among nurses. Nurs Manage [Internet]. 2019;27(5):1-5. https://www.doi.org /10.7748/nm.2019.e1820 
2. Kikanloo A, Jalali K, Asadi Z, Shokpour N, Amiri M, Bazrafkan L. Emotional intelligence skills: is nurses stress and professional competence related to their emotional intelligence training? a quasi experimental study. J Adv Med Prof [Internet]. 2019;7(3):138-143. https://www.doi.org/10.30476/JAMP.2019.74922

3. Szczygiel D, Mikolajczak M. Emotional intelligence buffers the effects of negative emotions on job burnout in nursing. Front. Psychol [Internet]. 2018;9(2649):1-10. https://www.doi.org/10.3389/fpsyg.2018.02649.

4. Goleman, D. Inteligência emocional. 13a ed. Lisboa: Temas e Debates; 2009.

5. Meng L, Qi J. The Effect of an emotional intelligence intervention on reducing stress and improving communication skills of nursing students. NeuroQuantology [Internet]. 2018;16(1):37-42. https://www.doi.org/10.14704/nq .2018.16.1.1175

6. Silva M, Queirós C, Cameira M, Vara N, Galvão A. Burnout e engagement em profissionais de saúde do interiornorte de Portugal. Psicol. Saúde Doenças [Internet]. 2015;16(3):286-299. https://www.doi.org/10.15309/15p $\operatorname{sd} 160302$

7. Najafpour J, Keshmiri F, Rahimi S, Bigdeli Z, Niloofar P, Homauni A. Effect of emotional intelligence on the quality of nursing care from the perspectives of patients in educational hospitals. J Patient Saf Qual Imp [Internet]. 2020;8(1):37-43. https://www.doi.org/10.22038/psj.2020.41216.1233

8. Huang H, Liu L, Yang S, Cui X, Zhang J, Wu H. Effects of job conditions, occupational stress, and emotional intelligence on chronic fatigue among Chinese nurses: a cross-sectional study. Psychol. Res. Behav. Manag [Internet]. 2019;12:351-360. https://www.doi.org/10.2147/PRBM.S207283

9. Lima P, Sabino K, Gouveia M, Avelino F, Fernandes M. Fatores estressores e as estratégias de enfrentamento utilizadas por enfermeiros hospitalares: revisão. Investig. Enferm. Imagen Dessar [Internet]. 2015;17(2):13-100. https:// www.doi.org//10.11144/Javeriana.ie17-2.feea

10. Pinto D, Santos M, Pires R. Relevance of indicators of clinical supervision strategies in nursing. Rev. Ren [Internet]. 2017;18(1):19-25. https://www.doi.org/10.15253/2175-6783.2017000100004

11. Giménez-Espert M, Prado-Gascó V, Valero-Moreno S. Efeito dos aspectos trabalhistas na comunicação, inteligência emocional e empatia em enfermagem. Ver. Latino-Am. Enfermagem [Internet]. 2019;27:e3118. https://www.d oi.org/10.1590/1518-8345.2933.3118

12. Santana V, Gondim, S. Regulação emocional, bem-estar psicológico e bem-estar subjetivo. Estud. Psicol (Natal) [Internet]. 2016;21(1):58-68. https://www.doi.org/10.5935/1678-4669.20160007

13. Ghamar M, Shamsolmolok J, Mohammad F. The effect of emotional intelligence on communication skills of nursing students. IJPN [Internet]. 2019;7(1):26-32. https://www.doi.org/10.21859/ijpn-07014

14. Ishii S, Horikawa E. The emotional Intelligence of japanese mental health nurses. Front. Psychol [Internet]. 2019; 10(2004):1-6. https://www.doi.org/10.3389/fpsyg.2019.02004

15. Paranhos R, Filho D, Carvalho da Rocha E, Júnior J, Freita D. Uma introdução aos métodos mistos. Sociologias. 2016;42(18):384-411. https://www.doi.org/10.1590/15174522-018004221

16. Martins C. Manual de análise de dados quantitativos com recurso ao IBM SPSS. Braga: Psiquilíbrios Ediçõos; 2011.

17. Marôco J. Análise Estatística com o SPSS Statistic. 7a ed. Pêro Pinheiro (Portugal): ReportNumber; 2018.

18. Elo S, Kyngäs H. The qualitative content analysis process. J. Adv. Nurs [Internet]. 2008; 62(1):107-115. https://www.doi.org/10.1111/j.1365-2648.2007.04569.x

19. Ribeiro J. Investigação e Avaliação em Psicologia e Saúde. $2^{a}$ ed. Lisboa: Placebo Editora; 2010.

20. Rodrigues N, Rebelo T, Coelho J. Adaptação da Escala de Inteligência Emocional de Wonge Law (WLEIS) e análise da sua estrutura factorial e fiabilidade numa amostra portuguesa. Psychologica [Internet]. 2011;55:189-207. https://www.doi.org/10.14195/1647-8606_55_10

21. Libbrecht N, Beuckelaer A, Lievens F, Rockstuhl T. Measurement invariance of the wong and law emotional intelligence scale scores: Does the measurement structure hold across far eastern and european Countries? Appl. Psychol [Internet]. 2014;63(2):223-237. https://www.doi.org/10.1111/j.1464-0597.2012.00513.x 
22. Keyes, C. Mental well-being: International contributions to the study of positive mental health. Dordrecht (Holanda): Springer; 2013.

23. Lamers S, Westerhof J, Bohlmeijer T, ten Klooster M, Keyes, C. Evaluating the psychometric properties of the Mental Health Continuum-Short Form (MHC-SF). J. Clin. Psychol [Internet]. 2011;67(1): 99-110. https://w ww.doi.org/10.1002/jclp.20741.

24. Fonte C, Silva I, Vilhena E, Keyes C. The portuguese adaptation of the mental health continuum-short form for adult population. Community Ment. Health J [Internet]. 2020; 56(2): 368-375. https://www.doi.org/10.100 7/s10597-019-00484-8

25. Ribeiro P, Honrado A, Leal I. Contribuição para o estudo da adaptação portuguesa das Escalas de Ansiedade, Depressão e Stress (EADS). Psicol. Saúde Doenças [Internet]. 2004;5(2):229-239. Disponible en: http://www .scielo.mec.pt/pdf/psd/v5n2/v5n2a07.pdf

26. Rahim M, Bonoma T. Managing organizational conflict: a model for diagnosis and intervention. Psychol. Rep [Internet]. 1979;44(3):1323-1344. https://www.doi.org/10.2466/pr0.1979.44.3c.1323

27. Leitão S, Cunha P. Manual de Gestão Construtiva de conflitos. $3^{a}$ ed. Porto: Edições Fernando Pessoa; 2016.

28. Takaki M, Sant'Ana D. A empatia como essência no cuidado prestado ao cliente pela equipe de enfermagem de uma unidade básica de saúde. Cogitare Enferm [Internet]. 2004;9(1): 79-83. https://www.doi.org/10.5380/ce .v9i1.1708

\section{Notas}

* Artículo original de investigación

\section{Licencia Creative Commons CC BY 4.0}

Cómo citar este artículo: Silva-Pereira AF, Santos-Prior AI, Ferreira M, Fonte C. Cuidar a los que se preocupan: efectividad de un programa de inteligencia emocional para enfermeros. Investig Enferm Imagen Desarr. 2020;22. https://doi.org/10.11144/Javeriana.ie22.cpep 